\title{
Aptamer Technology in the Targeted Delivery of Doxorubicin
}

\author{
Sarah E Sheikh ${ }^{1}$, Clay Marsh ${ }^{2}$ and Duaa Dakhlallah ${ }^{2 *}$ \\ ${ }^{1}$ University of Leeds, M Sc Biotechnology, UK \\ ${ }^{2}$ West Virginia University, USA
}

*Corresponding author: Duaa Dakhlallah, Research Assistant professor, West Virginia University, USA

Submission: 进 July 31, 2017; Published: 眥 September 05, 2017

\begin{abstract}
Thisreview aims at giving an insight at some of the recent approaches in using the aptamer technology in formulating the chemotherapeutic agent, doxorubicin, so as to achieve its targeted delivery and reduce its non-specific cytotoxicity.

Abbreviations: SELEX: Systematic Evolution Of Ligands By Exponential Enrichment; DOX: Doxorubicin; PSMA: Prostate-Specific Membrane Antigen; EpCAM: Epithelial Cell Adhesion Molecule; NPs: Nanoparticles; SPIONs: Superparamagnetic iron oxide nanoparticles; MRI: Magnetic Resonance Imaging; QDs: Quantum Dots; MUC1: Mucin 1; PSMA: Anti-Prostate Specific Membrane Antigen; TLC: Thermally Cross-Linked
\end{abstract}

\section{Introduction}

Aptamers are short DNA or RNA sequences that mimic antibodies in their high affinity and specificity to their targets, owing to their unique three-dimensional structure Catuogno [1]; Plourde et al. [2]. These targets may range from small molecules up to whole tissues Plourde et al. [2]. Compared to antibodies, aptamers exhibit improved properties such as lower immunogenicity, higher thermal stability, rapid and large-scale synthesis, and lower production costs Plourde et al. [2]. The process of aptamer evolution and selection is achieved using systematic evolution of ligands by exponential enrichment (SELEX) technology Sampson [3].

Doxorubicin (DOX) is an anthracycline-based chemotherapeutic agent that causes cancer cell death through intercalation into DNA, preferentially binding to double-stranded 5'-GC-3' or 5'-CG3 ' sequences, thus disrupting replication and transcription Meng et al [4]; Catuogno [1]. DOX is widely used owing toits efficacy in fighting a wide range of cancers such as carcinomas, sarcomas and haematological cancers Carvalho et al. [5]. Owing to its limited solubility and poor non-selective biodistribution, treatment with DOX often results in severe damage to healthy tissue Meng et al [4]. It was found to preferentially accumulate in the heart causing lifethreatening cardiotoxicity, in addition to inducing cytotoxicity in the brain, liver, and kidneys Carvalho et al. [5]; Tacar et al. [6].

Studies in drug formulations showed that conjugating aptamers to DOX reduced its cytotoxicity towards non-target cells both in vitro and in vivo, while maintaining a similar potency towards target cancer cells as the free drug Catuogno [1].

\section{Aptamer-Dox Direct Conjugation}

Due to the mechanism of action of DOX, its direct intercalation into aptamers was possible using a simple direct conjugation strategy without the need for any chemical modifications Catuogno
[1]. DOX was physically conjugated to multiple aptamers achieving successful cellular uptake and selective delivery to aptamer target positive cells, and therefore enhanced cytotoxicity of target cells and reduced toxicity of negative cells.

DOX-physical conjugates were formulated using A10 RNA aptamer, which binds to the prostate-specific membrane antigen (PSMA) Bagalkot et al. [7]. The RNA aptamer EpDT3 specific against the cancer stem cell marker, epithelial cell adhesion molecule (EpCAM), was conjugated to DOX, and the conjugate achieved selective delivery of the drug and enhanced cytotoxicity to retinoblastoma cells, as compared to non-cancerous Müller glial cells Subramanian et al. [8]. DOX was also directly conjugated to MA3 DNA aptamer specific to Mucin 1 (MUC1) protein, which is overexpressed in most adenocarcinomas $\mathrm{Hu}$ et al. [9]. It was also conjugated to an anti-HER2 DNA aptamer, where the complex was found to preferentially bind to HER2-positive but not HER2negative breast cancer cells Liu et al. [10].

AS1411 is a guanine-rich DNA aptamer that forms a stable G-quadruplex structure to specifically target nucleolin, which is overexpressed on tumor cell surfaces Zhang et al. [11]. This aptamer was used by Trinh et al. [12] to develop a novel drug-DNA adduct, where it was cross-linked to DOX using formaldehyde, maintaining the binding affinity of the original aptamer, and achieving hepatocellular carcinoma targeting.

Multiple approaches were undertaken to improve the properties of the DOX-physical conjugates. Aiming to improve the properties of the DOX-physical conjugates, Meng et al [4]. Modified TLS11a, a DNA aptamer specific for human hepatocellular carcinoma cells, so as to include a long Guanine-Cytosine tail at its 5' end. This led to increasing available DOX binding sites, hence achieving a higher DOX-aptamer, and ultimately enhancing the killing efficiency of 
target cancer cells. In another attempt to broaden the range of the aptamer-conjugated DOX, two modified monovalent aptamers, sgc8c and sgd5a, were self-assembled to generate a bi-specific drug carrier with multiple drug loading sites for DOX. This bioconjugate showed bi-specific cell binding, drug delivery, and cytotoxicity in target cells in cell mixtures Zhu et al. [13].

\section{Nanoparticle-Based Dox Bioconjugates}

Among the advances that have been made in the past decades in the field of drug formulations is the use of nanoparticles (NPs) as carriers for targeted drug delivery. Their high surface-area-tovolume ratio allows delivering higher doses of drugs to target organs in a controlled release manner. In addition, they are suitable for administration of both hydrophilic and hydrophobic substances by various routes, they have improved biodistribution and preferential accumulation in tumors, using the unique pathophysiology of tumors Wang et al. [14]; Jabir et al. [15].

\section{Gold Nanoparticles}

Gold NPs are widely used in drug formulations owing to their favorable properties such as being non-toxic, biocompatible, highly stable, of small dimensions, and having a surface that can easily be functionalized to conjugate the targeting aptamers Catuogno [1].

Seeking to make use of these properties, Zhao et al. [16] managed to formulate a hollow gold nanosphere that was loaded with high payloads of doxorubicin (DOX). That surface of the sphere was chemically conjugated with RNA aptamers specific for CD30, a diagnostic biomarker for Hodgkin's lymphoma and anaplastic large cell lymphoma. The formed bioconjugate was found to be pH sensitive, releasing $80 \%$ of the loaded DOX within 2 hours at $\mathrm{pH}$ 5, thus attaining targeted delivery to cancer cells, and having a minimal effect on normal tissue.

Another group adopted using gold NPsto co-deliver the photosensitizer TMPyP4 in addition to DOX Shiao et al. [17]. They were physically attached to the surface of gold NPs conjugated with the anti-nucleolin aptamer AS1411. The results revealed superior toxicity towards targeted cells than individual therapies.

In an attempt to achieve dual targeting of both extracellular and intracellular receptors specific to tumors, DOX was immobilized on the surface of gold nanoclusters conjugated to the anti-nucleolin aptamer AS1411, in addition to a cyclic RGD specific to $\alpha v \beta$ 3integrins over-expressed on the surface of tumor tissues. This prodrug bioconjugate achieved enhanced tumor affinity, deep tumor penetration, and improved cytotoxic activity both in vitro and in vivo Chen et al. [18].

\section{Magnetic Nanoparticles}

Superparamagnetic iron oxide nanoparticles (SPIONs) are considered promising theranostic agents owing to facile modification of their surfaces to accept different targeting ligands, in addition to their magnetic properties which give thema diagnostic valuewhile using magnetic resonance imaging (MRI) technology Catuogno [1].
Making use of these properties, Wang et al. [14] managed to develop a theranostic system that was capable of targeting DOX to prostate-specific membrane antigen (PSMA)-positive prostate cancer cells, while functioning as an MRI contrast agent. This was obtained by using the targeting aptamer, A10, which was modified to bind to thermally cross-linked (TLC)-SPIONs. DOX was loaded by intercalation in the CG sequence aptamer and by charge interactions with TCL-SPIONs. Similarly, PSMA-positive prostate cancer cells were targeted using DOX-loaded SPIONs, using DNA-RNA hybrid A10-3-J1 PSMA specific aptamer Leach et al. [19]. DOX-loaded TCLSPIONs were also bioconjugated to the PSMA positive targeting aptamer A10 RNA as well as DUP-1 peptide aptamer specific to PSMA negative cells, thus attaining dual targeting of PSMA positive and negative prostate cancer cells Min et al. [20].

\section{Liposomes}

Liposomes are spherical phospholipid bilayered structures that have the advantage of being able to encapsulate hydrophilic drugs in their aqueous core, as well as hydrophobic drugs in their lipid bilayer Catuogno [1]. Attempting to use liposomes in targeted delivery of chemotherapeutics, Xing et al. [21] formulated DOX loaded liposomes, and functionalized them with the anti-nucleolin aptamer AS1411. The results of this study showed improved targeted antitumor efficacy both in vitro and in vivo. Another group Baek et al. [22] developed a novel aptamer-liposome complex called aptasome, in which the aptamer-conjugated micelles were inserted into a liposome.

Here they used the anti-prostate specific membrane antigen (PSMA) A9 RNA aptamer to functionalize the DOX-encapsulating aptamosomes. An interesting formulation was prepared by Chuang et al. [23], in which DOX,as well as gold nanocages and the bubble generating agent ammonium bicarbonate (ABC), were encapsulated into a liposome conjugated to the anti-MUC-1 aptamer. Upon NIR irradiation, the gold nanocages generate localized heat, leading to $\mathrm{ABC}$ decomposition and production of carbon dioxide bubbles, triggering the release of DOX.This spatially controllable laser irradiation allows reduced non-specific toxicity. In a recent study, Plourde et al. [2] designed a series of DNA aptamer sequences specific to DOX, displaying multiple binding sites and various binding affinities. These DOX-specific aptamers were able to drive the drug into liposomes, thus achieving aptamer specific properties and liposomal controlled release.

\section{Quantum Dots}

Quantum dots (QDs) are nanocrystals with unique optical properties which can be conjugated to targeting ligands to generate molecular biosensors for real-time tracking and imaging Catuogno [1]; Savla et al. [24] managed to formulate QDs bioconjugated with MUC1 specific aptamer, targeting ovarian cancer. DOX was attached to the QDs via $\mathrm{pH}$-responsive hydrazone bonds that undergo rapid hydrolysis in mildly acidic $\mathrm{pH}$, thus providing stability of the conjugate in the systemic circulation and drug release inside cancer cells. Confocal microscopy and in vivo imaging of the complex revealed its preferential accumulation in ovarian tumors, 
with higher cytotoxicity compared to the free drug.Aiming to achieve zero premature release in addition to targeted delivery of DOX, Zhang et al. [11] formulated a traceable and dual-targeted drug delivery system based on capping DOX loaded mesoporous silica-coated quantum dots with a DNA hybrid of the antisense oligonucleotides, antimiR-21, coupled at the 3' end of the AS1411 aptamer. This bioconjugate achieved aptamer guided targeted delivery via AS1411, and once inside the cell, antimiR-21 would bind by complementary base pairing to miR-21, which is overexpressed in various human cancers, activating caspase-dependent apoptosis. This competition of miR-21 to fully hybridize the anchored DNA triggers on-command release of DOX from the bioconjugate.

\section{Carbon Nanomaterials}

Single walled carbon nanotubes (SWNTs) represent a promising tool for targeted drug delivery owing to the ease of their enhanced cell membrane penetrability by clathrin-mediated endocytosis, high drug loading capacity, tunable bio-distribution and pharmacokinetic properties achieved by controlling the size, the surface chemistry, and the targeting groups. In addition, they exhibit good biocompatibility, water solubility, low-toxicity following appropriate functionalization Meng et al. [25]. Employing such properties, Yang et al. [26] developed SWNTs encapsulating DOX and conjugated to sgc8 aptamers that were caged by the hybridization to complementary DNA strands. Upon exposure to near infrared (NIR) laser, the two strands of the DNA aptamer dehybridize, allowing sgc8 to specifically recognize target cells. The dual-targeting capability and spatial specificity of this technique allow reduced non-specific toxicity.

In summary, multiple approaches for doxorubicin targeted drug delivery have been developed using aptamers as targeting agents. Most of these studies have shown successful selective delivery of doxorubicin to targeted cancer cells and decreased non-specific cytotoxicity, in both in vitro and in vivo contexts.

\section{References}

1. Catuogno S, Esposito CL, DE Franciscis V (2016) Aptamer-Mediated Targeted Delivery of Therapeutics: An Update. Pharmaceuticals (Basel) $9(4)$.

2. Plourde K, Derbali RM, Desrosiers A, Dubath C, Vallee-Belisle A et al. (2017) Aptamer-based liposomes improve specific drug loading and release. J Control Release 251: 82-91.

3. Sampson T (2003) Aptamers and SELEX: the technology. World Patent Information 25: 123-129.

4. Meng L, Yang L, Zhao X, Zhang L, Zhu H, et al. (2012a) Targeted delivery of chemotherapy agents using a liver cancer-specific aptamer. PLoS One 7: e33434.

5. Carvalho C, Santos RX, Cardoso S, Correia S, Oliveira PJ, et al. (2009) Doxorubicin: the good, the bad and the ugly effect. Curr Med Chem 16: 3267-3285.

6. Tacar 0, Sriamornsak P, Dass CR (2013) Doxorubicin: an update on anticancer molecular action, toxicity and novel drug delivery systems. J Pharm Pharmacol 65: 157-70.

7. Bagalkot V, Farokhzad OC, Langer R, Jon S (2006) An aptamer-doxorubicin physical conjugate as a novel targeted drug-delivery platform. Angew Chem Int Ed Engl 45: 8149-8152.
8. Subramanian N, Raghunathan V, Kanwar JR, Kanwar RK, Elchuri SV, et al. (2012) Target-specific delivery of doxorubicin to retinoblastoma using epithelial cell adhesion molecule aptamer. Mol Vis 18: 2783-2795.

9. Hu Y, Duan J, Zhan Q, Wang F, LU X, et al. (2012) Novel MUC1 aptamer selectively delivers cytotoxic agent to cancer cells in vitro. PLoS One, 7(2): e31970.

10. Liu Z, Duan JH, Song YM, MA J, Wang FD, et al. (2012) Novel HER2 aptamer selectively delivers cytotoxic drug to HER2-positive breast cancer cells in vitro. J Transl Med 10: 148.

11.Zhang P, Cheng F, Zhou R, Cao J, Li J, et al. (2014) DNA-hybrid-gated multifunctional mesoporous silica nanocarriers for dual-targeted and microRNA-responsive controlled drug delivery. Angew Chem Int Ed Engl 53: 2371-5.

12.Trinh TL, Zhu G, Xiao X, Puszyk W, Sefah K, et al. (2015) A Synthetic Aptamer-Drug Adduct for Targeted Liver Cancer Therapy. PLoS ONE 10: e0136673.

13.Zhu G, Meng L, Ye M, Yang L, Sefah K, et al. (2012) Self-assembled aptamer-based drug carriers for bispecific cytotoxicity to cancer cells. Chem Asian J 7: 1630-1636.

14.Wang AZ, Bagalkot V, Vasilliou CC, GU F, Alexis F, et al. (2008) Superparamagnetic iron oxide nanoparticle-aptamer bioconjugates for combined prostate cancer imaging and therapy. ChemMedChem 3: 1311-1315.

15.Jabir NR, Tabrez S, Ashraf GM, Shakil S, Damanhouri GA et al. (2012) Nanotechnology-based approaches in anticancer research. International Journal of Nanomedicine 7: 4391-4408.

16.Zhao N, You J, Zeng Z, Li C, Zu Y (2013) An ultra pH-sensitive and aptamer-equipped nanoscale drug-delivery system for selective killing of tumor cells. Small 9: 3477-3484.

17.Shiao YS, Chiu HH, WU PH, Huang YF (2014) Aptamer-functionalized gold nanoparticles as photoresponsive nanoplatform for co-drug delivery. ACS Appl Mater Interfaces 6(24): 21832-21841.

18.Chen D, Li B, Cai S, Wang P, Peng S, et al. (2016) Dual targeting luminescent gold nanoclusters for tumor imaging and deep tissue therapy. Biomaterials 100: 1-16.

19.Leach JC, Wang A, Ye K, Jin S (2016) A RNA-DNA Hybrid Aptamer for Nanoparticle-Based Prostate Tumor Targeted Drug Delivery. Int J Mol Sci 17: 380 .

20. Min K, JO H, Song K, Cho M, Chun YS, et al. (2011) Dual-aptamer-based delivery vehicle of doxorubicin to both PSMA (+) and PSMA (-) prostate cancers. Biomaterials 32(8): 2124-2132.

21.Xing H, Tang L, Yang X, Hwang K, Wang W, et al. (2013) Selective Delivery of an Anticancer Drug with Aptamer-Functionalized Liposomes to Breast Cancer Cells in Vitro and in Vivo. J Mater Chem B Mater Biol Med 1(39): 5288-5297

22. Baek SE, Lee KH, Park YS, OH DK, OH S, et al. (2014) RNA aptamerconjugated liposome as an efficient anticancer drug delivery vehicle targeting cancer cells in vivo. J Control Release 196: 234-242.

23. Chuang EY, Lin CC, Chen KJ, Wan DH, Lin KJ, et al. (2016) A FRET-guided, NIR-responsive bubble-generating liposomal system for in vivo targeted therapy with spatially and temporally precise controlled release. Biomaterials 93: 48-59.

24.Savla R, Taratula O, Garbuzenko O, Minko T (2011) Tumor targeted quantum dot-mucin 1 aptamer-doxorubicin conjugate for imaging and treatment of cancer. J Control Release 153: 16-22.

25.Meng L, Zhang X, LU Q Fei Z, Dyson PJ (2012b) Single walled carbon nanotubes as drug delivery vehicles: targeting doxorubicin to tumors. Biomaterials 33: 1689-98.

26. Yang Y, Liu J, Sun X, Feng L, Zhu W, et al. (2016) Near-infrared lightactivated cancer cell targeting and drug delivery with aptamer-modified nanostructures. Nano Research 9: 139-148. 